\title{
AIRBORNE SEPARATION ASSURANCE AND TRAFFIC MANAGEMENT: RESEARCH OF CONCEPTS AND TECHNOLOGY
}

\author{
Mark G. Ballin, ${ }^{*}$ David J. Wing, Monica F. Hughes, and Sheila R. Conway \\ NASA Langley Research Center \\ Hampton, Virginia
}

\begin{abstract}
$\underline{\text { Abstract }}$
To support the need for increased flexibility and capacity in the future National Airspace System, NASA is pursuing an approach that distributes air traffic separation and management tasks to both airborne and ground-based systems. Details of the distributed operations and the benefits and technical challenges of such a system are discussed. Technology requirements and research issues are outlined, and NASA's approach for establishing concept feasibility, which includes development of the airborne automation necessary to support the concept, is described.
\end{abstract}

\section{$\underline{\text { Introduction }}$}

A significant portion of the aviation user community has identified a need for increasing flexibility of aircraft operations while retaining guaranteed separation from hazards. This need has been expressed as a new operational paradigm, "free flight," which reduces reliance on centralized air traffic management. Free flight is defined as a safe and efficient flight operating capability under instrument flight rules in which operators have the freedom to select their path and speed in real time. ${ }^{1}$ Air traffic restrictions are only used to ensure separation and safety of flight, preclude exceeding airport capacity, and prevent unauthorized flight through special use airspace. Proponents of free flight are concerned that the current system of air traffic management imposes a substantial handicap on the user community, which is forced to rely on an infrastructure that is extremely difficult to modernize due to its complexity and size. They believe free flight will enable future growth that would otherwise be constrained by a centralized ground-based system. Many also feel that competition-related business-case

Copyright (C) 1999 by the American Institute of Aeronautics and Astronautics, Inc. No copyright is asserted in the United States under Title 17, U.S. Code. The U.S. Government has a royalty-free license to exercise all rights under the copyright claimed herein for Government purposes. All other rights are reserved by the copyright owner.

*Senior Member, AIAA decisions are difficult to make due to restrictions inherent in the current system. Therefore, free flight has a goal not only to optimize the system, but also to open the system up to allow each user to self-optimize. ${ }^{1}$

One approach to achieving the long-term goal of mature-state free flight is to define a type of airspace that would allow aircraft to manage their operations autonomously. ${ }^{2,3}$ This approach would require an aircraft to have autonomous separation assurance capability to gain access to "free flight airspace," which would most likely be defined as en route airspace above a specified flight level. This may not be appropriate for the continental United States airspace (CONUS), where it is imperative to minimize equipment required for airspace access and allow for a mix of avionics capability in individual aircraft. In addition, the approach would not enable free flight operations outside free flight airspace where limits to traffic capacity frequently occur, such as regions where aircraft must merge and sequence as they transition to terminal airspace. An alternative approach to achieving mature-state free flight is to distribute capability and responsibility for traffic management between aircraft and ground-based air traffic control over as much airspace as possible, while mandating as few equipage requirements for airspace access as possible.

Such an approach is referred to herein as distributed air/ground traffic separation and management (DAG TM), and is the subject of study by the National Aeronautics and Space Administration (NASA) as part of its Advanced Air Transportation Technologies Project (AATT). DAG TM is based on the fundamental premise that all system participants can be used both as information suppliers and as users, thereby enabling user collaboration and cooperation in all levels of traffic management decision making. DAG TM addresses traffic separation and traffic management over short event horizons, involving decision making by flight crews and air traffic service providers (which include controllers and traffic management coordinators). DAG TM also incorporates strategic planning and fleet management over longer event horizons, involving the 
triad of the flight crew, the service provider, and airline operational control (AOC). In collaboration with industry, the international R\&D community, and other NASA programs, the AATT project will investigate technical feasibility of DAG TM. In support of the feasibility assessment, NASA will develop operational concepts and enabling technology.

This paper provides an overview of NASA's plans for investigation of DAG TM for traffic separation and local flow management in en route airspace, with an emphasis on airborne separation assurance. Potential benefits and technical challenges are summarized, and an operational concept currently under investigation is described. A discussion of plans for research and technology development is also included, followed by a description of the airborne technologies required to enable the concept.

\section{$\underline{\text { DAG TM Benefits }}$}

DAG TM is anticipated to have many benefits over the current centralized mode of air traffic management and operations. Potential benefits include increased safety, increased user flexibility and user efficiency, global interoperability, and operational and economic scalability. Under DAG TM operations, assurance of safety through traffic separation will no longer be dependent on a single sector controller team. Today, a loss of situational awareness by that team can result in a loss of separation between aircraft. In a mature-state distributed system, the ground-based controller team and the flight crews of both aircraft involved must lose awareness to cause the same loss of separation.

Increased system safety through redundancy is possible, since the capability to resolve traffic conflicts will no longer reside only at a single air traffic control facility.

Increased user flexibility may result from the removal of traffic management restrictions that have evolved based on a centralized, ground-based communication, navigation, and surveillance (CNS) infrastructure and the abilities and availability of controllers to separate aircraft. User efficiency may be improved by decreasing minimum separation standards and the additional buffers that controllers typically add to them, and by reducing the need to operate on a route structure or at fixed altitudes. Users may also gain efficiency by applying their goals and preferences directly to flight management rather than relying on a controller either to make assumptions about user preferences or to accommodate user requests.

Another potential benefit of DAG TM is that of worldwide operability and increased separation assurance capability in less-developed regions of global airspace, since a large portion of the required CNS infrastructure travels with each aircraft.

The operational and economic scalability of DAG TM may ultimately provide the greatest benefit. Current operations, which rely on centralized air traffic management, may not scale linearly with the anticipated growth of air traffic. In the future, either a controller team will be required to handle an increasing number of aircraft, or the team will be responsible for a smaller region of airspace. Even with improved ground-based automation, either of these solutions may rapidly approach limits of feasibility as both would require increases of controller attention, coordination, liaison, and manual tasks such as handoffs. ${ }^{4}$ An assessment of the capability for decision-aiding technology to improve controller workload management in the current mode of NAS operations concluded only marginal benefits may be attainable. According to Andrews, "Reasonable assumptions regarding the amount of workload relief provided by decision-support tools (for controllers) lead to the conclusion that only limited growth can be accommodated by this means." Under the DAG TM paradigm, each aircraft that enters a volume of airspace brings additional capabilities in both decision support automation and human decision making, thereby providing the potential to grow proportionately with an increase in throughput. Much higher traffic throughput levels may be attainable before limits are reached. A higher aircraft-to-controller ratio may be also possible, thereby keeping service provider costs within bounds as air traffic grows. Since capital and recurring costs of infrastructure would be more distributed, modernization and maintenance costs of the air traffic system may also be less cumbersome than they are today.

\section{Operational Environments}

En Route CONUS airspace can be divided into three major problem domains by traffic complexity: unconstrained airspace, constrained airspace, and transitional airspace. To be operationally viable, DAG TM may be required to work in all of these environments. Most research into free flight concepts to date applies to unconstrained airspace, which consists entirely of unconstrained aircraft that are not affected by traffic management or other highly dynamic localized constraints. Such environments are typically found during good weather in the western United States, away from major terminals, and can be expected to occur even less frequently as air traffic demand increases. Constrained airspace is characterized as containing dynamically changing regions that are either 
restricted or contain hazards to air traffic, such as severe convective weather cells. Transitional airspace is an environment that contains aircraft affected by flow constraints into terminal areas. Often thought of as the complex and dense traffic areas surrounding Class B airspace, transitional airspace can also occur many hundreds of miles from terminals as flow constraints back up into upstream Air Route Traffic Control Centers (ARTCC). A mix of unconstrained aircraft and many highly constrained aircraft in climb and descent phases of flight characterizes this environment. Aircraft transitioning to terminal airspace are often required to adhere to spatial crossing constraints, such as speed and altitude restrictions at facility boundaries, and temporal crossing constraints such as a required times of arrival (RTA) resulting from arrival metering, while simultaneously descending from cruise altitudes. In addition, all of the problems found in constrained airspace may also occur in transitional airspace. Terminal operations characterized by lower traffic density, such as Class B or C airspace in offpeak periods, share many qualities with transitional en-route airspace, and similar DAG TM solutions may apply.

The operational environments in which DAG TM must work are depicted with examples of typical problems in Figure 1. In constrained airspace, flight crews and/or controllers must:

- use the best available information to plan across multiple sectors and multiple facilities

- maintain separation with other aircraft, which may have significantly different performance and navigation capabilities

- maintain passenger safety and comfort by avoiding hazards such as severe convective weather and clear air turbulence

- avoid bottlenecking while rerouting about weather and special use airspace

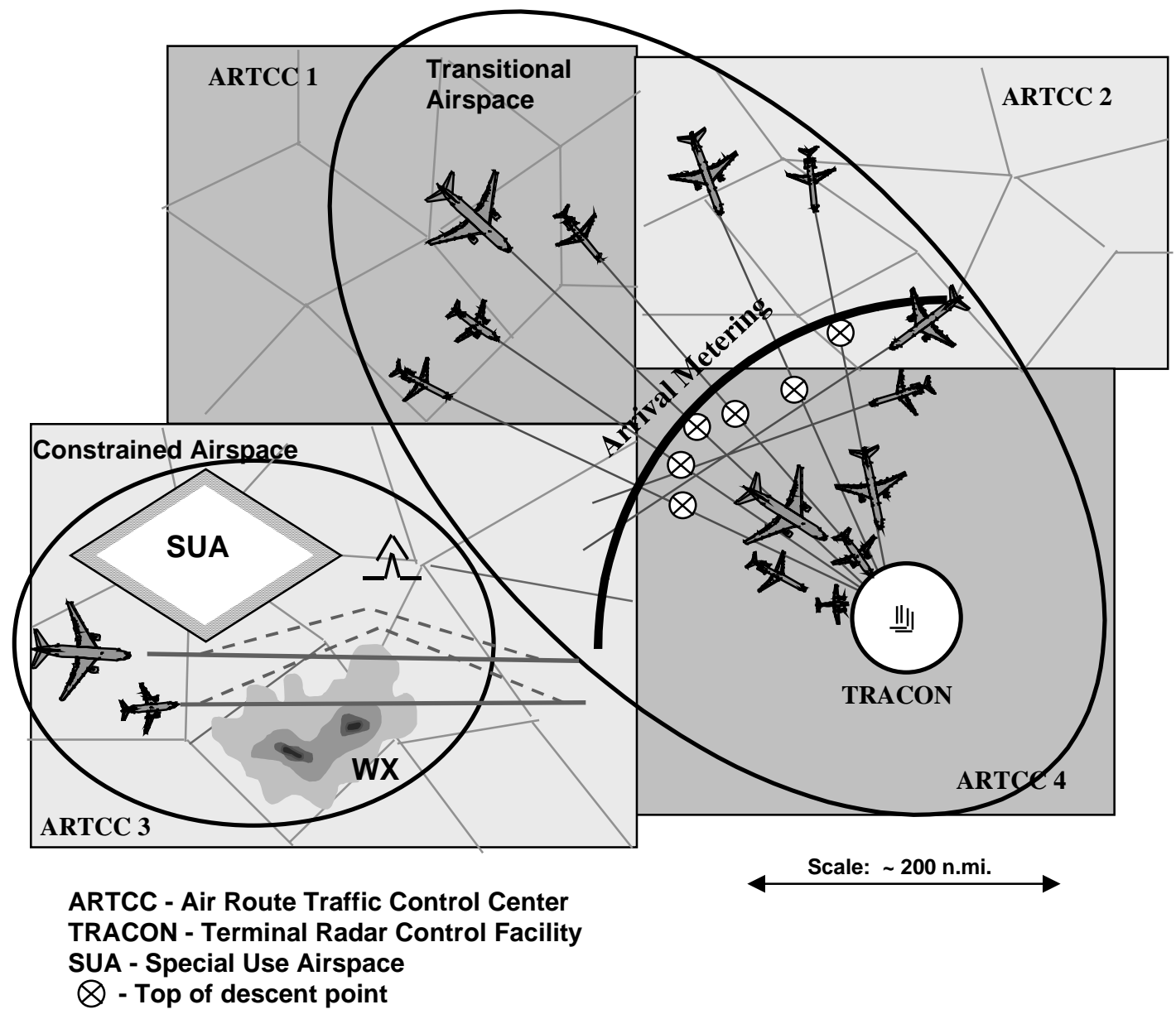

Figure 1. En route operational environments 
In addition to these tasks, transitional airspace requires crews and controllers to:

- comply with flow management constraints across facility boundaries or fixes

- $\quad$ descend as efficiently as possible while maintaining separation from crossing aircraft

- comply with terminal flow management constraints (such as in-trail spacing or metering)

- $\quad$ merge and sequence to maximize terminal airspace and runway utilization

\section{Concept of Operations}

NASA is developing a mature-state concept of operations for DAG TM in which the roles and responsibilities of human operators within the system are defined. The concept must be feasible and viable in constrained and transitional airspace, at least as safe and robust as the current system, and applicable to all user classes under all conditions. In developing the concept, mature-state requirements must be balanced against near-term National Airspace System (NAS) needs, and a clear path for transition from current operations to a modernized system must be defined.

NASA has been using two parallel approaches to concept development, referred to herein as requirements-based and capabilities-based. The requirements-based approach assumes advanced technology is available and can be implemented. It places heavy emphasis on user airborne independence desires, and is therefore designed to achieve a high degree of user self-determination for both infrastructure modernization and mature-state operations. The approach relies on the future existence of an advanced telecommunications network, a real-time interactive NAS-wide information system, air-to-ground data links, and air-to-air data link with capabilities greater than the minimum standards currently defined for Automatic Dependent Surveillance - Broadcast (ADS-B). The requirements-based concept is discussed below.

The technology projected to be available and the need for evolutionary modernization drives the capabilitiesbased approach, which is compliant with Federal Aviation Administration (FAA) modernization plans for the year $2015^{6,7}$ and standards documents such as the ADS-B minimum aviation system performance standards. ${ }^{8}$ The capabilities-based approach places a heavy emphasis on near-term user and system needs and certification requirements. Although lower benefits are expected from this approach relative to the requirements-based approach, the former may define a necessary evolutionary step in NAS modernization. A description of the capabilities-based concept can be found in Ref. 9.

Note that these DAG TM concepts may not be appropriate for busy terminal areas where airspace throughput goals dominate user flexibility and efficiency goals. Arrival and departure procedures that maximize runway utilization may be more appropriate in the terminal area, such as separation of aircraft into separate arrival streams based on their speed, and use of automation that balances loads among several runways. Advanced DAG TM concepts such as airborne selfspacing are under investigation, but are beyond the scope of this paper.

\section{Distributed Responsibility for Separation Assurance}

Conflicts are defined as predicted violations of separation between two or more aircraft as well as between an aircraft and other hazards such as severe weather or special use airspace (SUA). Autonomous airborne separation assurance is designed to provide each flight crew with the capability to detect conflicts and a means to alter their aircraft's trajectory to avoid them. Separation is compromised when an aircraft's protected zone, defined as one half of the minimum allowed horizontal and vertical distance between two aircraft, has been penetrated by the protected zone of another. Figure 2 illustrates the projected penetration of two protected zones and a potential conflict resolution.
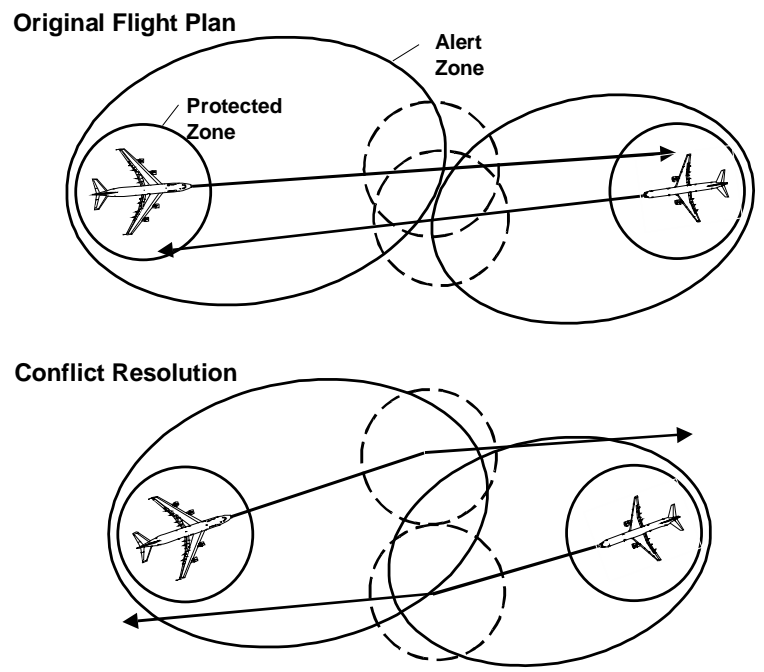

Figure 2. Airborne separation assurance

The sooner a conflict is detected, the greater the possibility of coordinating the ensuing resolution with flight planning goals. For conflicts detected with little notice, flight planning goals may be set aside in the interest of safety. Conflict resolution strategies are therefore a function of event horizon, which is defined 
as the time remaining before a near mid-air collision (NMAC) or a violation of SUA occurs. The optimum time for resolution is defined by the trade-off between certainty of prediction of a given conflict and the benefits to be gained from early resolution. As the point-of-conflict approaches, the probability of conflict increases while maneuver efficiency decreases. Paielli found the optimum event horizon for resolution to be roughly 15 minutes, based on aircraft information from ground-based radar surveillance and assuming that benefits of early resolution are only in maneuver efficiency. ${ }^{10}$ With improvements to position measurement accuracy, knowledge of flight crew intent, and by accounting for traffic management efficiency in determination of the time-to-resolve, resolution event horizons on the order of 30 minutes may be beneficial.

A potential distribution of responsibility between flight crews and controllers as a function of time to point-ofconflict is presented in Figure 3. Several temporal zones are defined based on the concept that user and controller goals, and hence their resolution strategies, change as a function of time to point-of-conflict. The sizes and characteristics of these zones will be subjects of research.
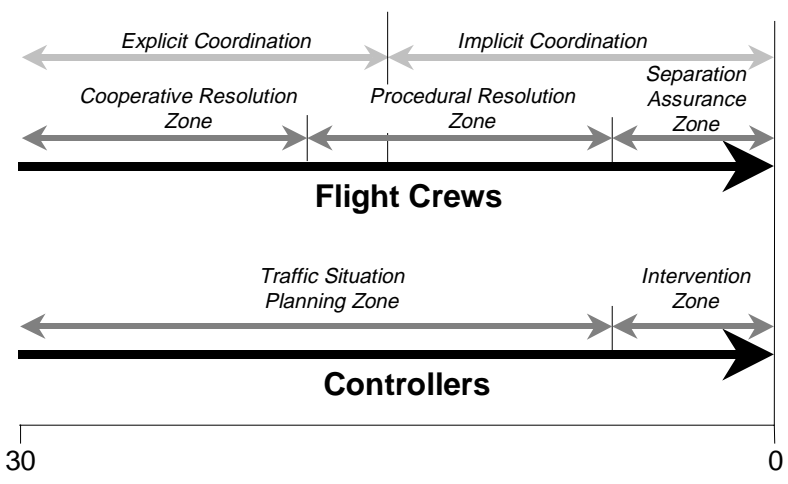

Time before NMAC, minutes (notional)

Figure 3. Flight crew and controller temporal zones

For conflicts detected while in the cooperative resolution zone, explicit coordination is performed between crews of two or more aircraft that are predicted to violate separation minima. Appropriately equipped aircraft have the opportunity to resolve the conflict cooperatively and without participation by the controller. Aircraft state information, such as position and altitude, and intent information, such as upcoming trajectory-change points, are broadcast from each aircraft. Based on these data as well as knowledge of goals, performance, and the environment, airborne decision support automation provides the crews with specific maneuver advisories. Cooperative resolutions are based entirely on the flight management goals of the participants. One aircraft may provide the entire resolution maneuver or several aircraft may maneuver partially. Explicit coordination between aircraft takes place either by direct negotiation or by each crew observing the actions of the other.

Concurrently, controllers are in the traffic situation planning zone and use decision support automation to maintain awareness of the traffic situation. With this awareness, they may predict future regions of traffic complexity too great to enable safe intervention. One measure of traffic complexity might be the number of conflicts predicted to occur in a region. The automation provides advisories to reroute some aircraft away from a predicted high-complexity region, or if found necessary for concept feasibility, advises controllers to reclaim autonomous maneuvering authority from all aircraft.

If flight crews do not resolve the conflict within the time defined by the cooperative resolution zone, they enter a procedural resolution zone. At such a point, there is not enough time for negotiation, and aircraft are required to follow predetermined rules for resolving a conflict. The rules dictate which aircraft must maneuver and the maneuver degrees of freedom. They may be based on extensions of visual flight rules, as developed by Eurocontrol. $^{2}$ If the time to NMAC continues to decrease beyond a specific threshold, resolution strategies must be coordinated implicitly. For implicit coordination, all aircraft are required to use identical conflict detection and resolution algorithms. Assuming each flight crew has identical information, the advisories provided to each are compatible. During implicit coordination, crew goals and maneuver efficiency are secondary to safe conflict resolution.

In the distributed air/ground concepts under investigation, the goal in airborne separation assurance is to resolve potential conflicts before a controller needs to intervene. However, if the aircraft do not achieve a conflict resolution, they will enter the controller's intervention zone. Within the zone, whether the controller has the responsibility to intervene or just the option to intervene will be a subject of research. By intervening, the controller assumes responsibility for separation assurance and exercises positive control. The size of the zone is based on look-ahead practices and comfort levels of controllers. The controller is provided ground-based automation to assist in intervening and resolving the conflict by issuing clearances for one or more aircraft to maneuver. Concept feasibility depends on the intervention zone being a reasonable size. ${ }^{1}$

The flight crew's separation assurance zone corresponds to the controller's intervention zone, as shown in Figure 3. Airborne separation capability should be used 
to maximize safety in this zone, even though the controller may have responsibility for separation assurance. An extension of the current Traffic Alert and Collision Avoidance System (TCAS) concept of operations is used. In the separation assurance zone, crew goals, maneuver efficiency, and passenger comfort are secondary to safe conflict resolution or collision avoidance. To minimize the number of missed alerts, conflict detection is based on aircraft state information only. Resolution algorithms and crew advisories are designed for ownship safety at the expense of situational awareness. The crew is advised where not to go rather than being given an advisory consisting of a single trajectory. The National Aerospace Laboratory of The Netherlands (NLR) has developed and is investigating a system that may be appropriate for use in the separation assurance zone. ${ }^{3}$

\section{Operations in the DAG TM Environment}

The requirements-based concept is a modification of the Air Traffic Management Partnership concept developed by Lockheed-Martin under funding from NASA. ${ }^{11}$ The concept defines two fundamental aircraft equipage levels: independent and dependent. Independent aircraft are voluntarily equipped to meet all requirements for autonomous airborne separation assurance. Dependent aircraft do not meet all requirements and therefore need some level of separation assurance and traffic management from the ground-based controller. For airspace access, all aircraft must have the capability to broadcast their position.

Flight crews of independent aircraft have responsibility for separation assurance from other aircraft, hazardous weather, special use airspace, and terrain. They receive constraints such as RTAs from ground-based systems, and they have the authority to manage their trajectories to meet those constraints. Independent aircraft crews may change their trajectories without approval of the controller, but they must continually notify the controller and aircraft within broadcast range of their current intentions. The controller may impose flight guidance by exception at any time. As shown in Figure 2, the time-based alert zones defined in Ref. 1 surround each aircraft. For predicted conflicts, these alert zones define the controller's intervention zone. If alert zones of two aircraft touch and an unresolved conflict exists between them, the controller may be responsible for stepping in to resolve the conflict. As in the current system, responsibility for separation cannot be transferred unless the receiver of the responsibility agrees. Therefore, if the flight crew has separation responsibility, the controller is not required to accept responsibility if asked by the crew.
Figure 4 illustrates information transfer required for independent-aircraft conflicts. Using ADS-B, independent aircraft exchange aircraft state and intent information. If the aircraft are not within ADS-B range, traffic information is uplinked to each aircraft from a ground-based traffic information service (TIS), which uses downlinked trajectory information and all available sources of surveillance to determine aircraft position and intent. Using on-board decision-aiding systems, the aircraft jointly arrive at a cooperative or coordinated solution before their alert zones merge. They may agree to a resolution that allows them to merge, but the controller must be notified of the agreement before the zones touch. Notification is in the form of ADS-B broadcast or downlink for both aircraft's conflict-free time- and position-constrained (4-D) trajectory information. Independent aircraft whose alert zones merge without having notified the controller of a conflict resolution forfeit their independence for the duration of the encounter. The ground-based decisionaiding system assists the controller in monitoring for conflicts and determining constraints or trajectory restrictions. The system alerts the controller if the protected zones are predicted to merge, and generates conflict resolution advisories in the form of one or more modified trajectory change- points and/or crossing restrictions for transmittal by uplink or voice communication.

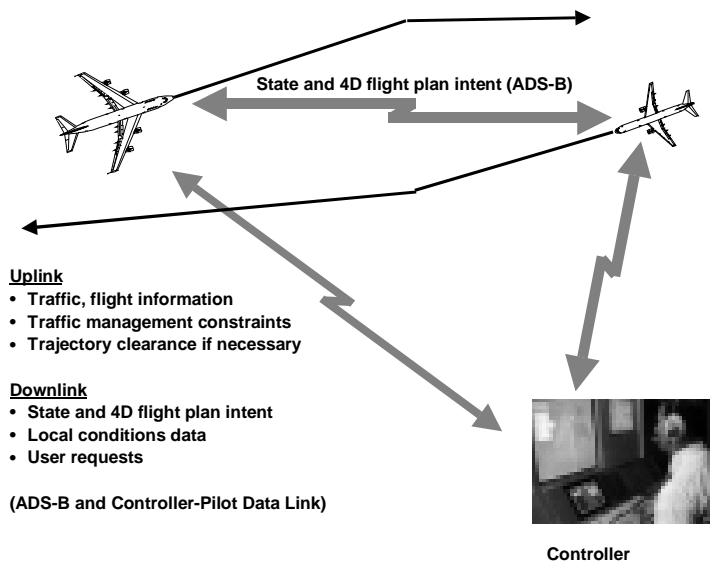

Figure 4. Information transfer between two independent aircraft and the controller.

The controller assures separation and manages traffic flow for dependent aircraft. Ground-based decisionsupport automation assists the controller in predicting and resolving conflicts and in negotiating with independent aircraft for a collaborative solution. When alert zones of independent and dependent aircraft merge, the ground-based automation advises the controller of resolution trajectories or maneuver limitations. If there is no controller intervention, independent aircraft will still have full capability for avoiding dependent aircraft. 
To prevent unintentional penalization to independent aircraft for having autonomous separation capability, the controller will give them priority when possible.

When operating in constrained and transitional airspace, dependent aircraft may need to be segregated from independent aircraft. Specific sectors may be designed and staffed to be capable of handling large numbers of dependent aircraft, thereby freeing the remaining airspace for independent aircraft.

\section{Enabling Decision Support Technology}

The requirements-based concept may require extensive airborne and ground-based automation with significant real-time data exchange. Required airborne functions consist of hazard detection, prioritization, notification, and resolution, and automated and provisional "what-if" planning for traffic management conformance. In addition, automated accommodation of flight crew or AOC goals and preferences and an advanced user interface will be necessary. Planned trajectories must consider aircraft performance and ride quality constraints, and must be compatible with flight management system 4-D guidance capabilities.

At a minimum, the service provider requires automation to assist in conflict-free planning integrated with local traffic management. The service provider may also need the capability to detect traffic and airspace complexity hazards and provide advisories for intervention, including rerouting of selected aircraft to reduce complexity. Automated resolutions may need to be based on policy issues, such as allowing independent aircraft to have priority. Sector controllers require automation to assist in conflict detection for all aircraft, resolution advisories based on controller goals and preferences for all aircraft, inter-sector coordination, and provisional planning. Traffic management coordinators require automated generation of dynamic traffic management constraints such as RTAs, altitude restrictions, or in-trail spacing restrictions. The accurate generation of these constraints is critical to system feasibility; not only must the RTAs be achievable within performance limits of the aircraft, but system throughput loads must be accurately predicted. If the constraints are not reliable, crews and controllers may attempt to gain advantage by arriving early.

\section{DAG TM Research}

Over the next several years, NASA free-flight research will focus on feasibility and potential benefits of DAG TM operations and the supporting decision aiding technology. DAG TM poses several challenges that must be addressed before its feasibility can be established. These challenges include distributed responsibility for separation assurance, distributed traffic management, human-automation interaction, and technology development.

\section{Distributed Responsibility for Separation Assurance}

Because control is distributed among several decision makers, DAG TM increases the risk of confusion over responsibility for separation assurance and the transfer of that responsibility. The RTCA vision of free flight states that the service provider retains overall responsibility for safe operation of the NAS. ${ }^{1}$ In Figure 3, several temporal zones before time of NMAC are proposed that define these roles and limits. Defining practical time horizons and limits of authority for the flight crew and controllers in these zones will be addressed through research.

By remaining continually cognizant of developing situations, controllers are able to manage complex traffic. When responsibility is transferred from ground to air or air to ground, the receiving individual may be hampered by not having been continually engaged. Research will address the situational awareness of pilots and controllers while engaged in DAG-TM operations, and the information required by each participant.

Other issues related to the topic of distributed responsibility will also be studied. Because of the inherent flexibility in a free-flight environment, there is a possibility of system abuse or disputes between decision makers. In addition, not all aircraft in the NAS will have the same self-separation capability. Research will address separation assurance of aircraft with mixed equipage and separation assurance from non-compliant equipped traffic. The necessity for direct negotiation between distributed decision makers (air-air or airground) will also be determined.

\section{Distributed Traffic Management}

In today's operations, meeting the simultaneous goals of separation assurance, expeditious traffic flow, and both implicit and explicit user preference accommodation requires a controller to devise, execute, and continually update a plan for managing traffic. By maintaining a perspective of the total traffic situation, the controller also assures that the overall traffic complexity does not exceed the limits of safety. The problem of distributing planning and execution of traffic management among many decision makers while meeting the goals above may ultimately define the limits of feasibility of DAG TM. At a minimum, any feasible concept must account for communication of intent information between planners and negotiation of solutions to conflicting 
plans. This problem will be examined in increasingly complex environments, ultimately addressing real-world issues of dynamically changing airspace constraints, transition traffic (ascents/descents), and changing wind fields. Through simulation with increasingly complex models, feasibility and viability of DAG-TM concepts in the presence of such uncertainties will be determined.

\section{Human-Automation Interaction}

The ability of the DAG-TM system operators to function with consistency throughout the complexities of the real-world operational environment will be pivotal to safe and efficient operations. Controller workload management will be an issue in light of predicted air traffic growth. Flight crew workload increases cannot be tolerated. Decision-aiding automation will benefit both controllers and flight crews in providing diligent monitoring of dynamic situations and advisories where appropriate. The reduction in clearances is projected to significantly offset the workload associated with the additional tasks. NASA research will include a focus on workload of flight crews and service providers in the DAG-TM environment. Both workload and the ability of human operators to successfully perform their tasks are closely dependent on the design of decision-aiding automation and associated interfaces.

\section{Technology Development}

To enable the technical feasibility of DAG TM, NAS infrastructure research and development will be necessary. Standards for some components of the future NAS have already begun to evolve without a concept of mature-state DAG-TM operations to substantiate the requirements. Conceptual-level research will address the CNS technologies required for DAG-TM operations. Examples of research topics to be addressed include determining necessary ADS-B performance and message content, position accuracy requirements, and data-link communications performance.

Many research issues are associated with the development of the airborne technology necessary for autonomous operations. NASA will develop the airborne technology necessary for DAG TM operations, addressing these development research issues in parallel with feasibility research. Airborne technology is discussed in the Airborne Automation Development section that follows.

The ground component of a distributed system will require decision-aiding automation as well. Tools designed for the current operational environment will probably not be sufficient for the entire task. Given the redistribution of responsibilities in DAG TM and the need for extensive air-ground coordination in a traffic environment with mixed-capability aircraft, the service provider will require decision-aiding automation designed specifically for DAG TM. Research will identify functional requirements for such a system as needed to coordinate with the airborne automation.

\section{Research Approach}

Experiments will be conducted in three venues: a newly developed, flexible workstation-based simulation; highfidelity flight deck simulation; and in flight. To perform basic operational and system-wide distributed traffic management research, a robust, mid-fidelity, workstation-based research simulation environment referred to as Free Flight Simulation (FFSim) is being developed. ${ }^{12}$ The FFSim environment will be used for concept-level operational feasibility assessments, system-level requirements definition, airborne and CNS technology requirements determination, and basic human-centered design and assessment. The highfidelity simulation environment will be used for investigations of human performance in a distributedresponsibility environment and in human-automation interaction studies. This environment will be appropriate when realistic setting or taskload representation is germane to the research topic. Finally, flight testing will be used to validate simulation models, assumptions, and some conclusions, and to enable the demonstration of airborne technologies and operations.

FFSim is designed to provide a testbed for investigations of DAG-TM operational concepts and their associated pilot/controller decision support tools. The simulation has interchangeable components including models of aircraft systems, aircraft performance, ground-based air traffic control systems, and a suite of CNS infrastructure technologies. The FFSim elements are modeled at a level of sophistication sufficient for determining feasibility and initial quantitative benefits. Interfaces to pilot and controller subjects are not intended to duplicate the workload or environment of certifiable systems. Rather, the interfaces are intended to enable pilot and controller interactions with decision aids and with each other to plan and execute flight operations.

To complement research using human subjects, a batchmode capability will be added to FFSim. In batch mode, automated human-performance models replace actual human participants. This capability will enable very large numbers of aircraft encounters, thereby allowing quantification of system sensitivities to stochastic factors such as crew response time and wind prediction accuracy. System design issues such as trajectory adherence requirements or the need to rely on performance models for target aircraft trajectory 
prediction will be investigated as well. In addition, batch simulations will enable benefit assessment and determination of the impact of reducing separation standards and other restrictions on overall system safety.

As the concept-level research and the airborne decisionaiding automation progress, studies will begin to make use of high-fidelity simulations. A realistic, full mission cockpit will be required for some of the operatoroperator and operator-system interaction studies. Highfidelity simulations will enable research in procedures, operator workload, crew resource management, and assessing the impact of the new technology on crew situational awareness. In this venue, it will be possible to conduct experiments requiring greater control of environment than can be achieved in flight. Highfidelity simulation will also be necessary for final verification of new airborne technology prior to flight.

Flight tests will be performed for validation of simulation models, assumptions, and some conclusions and for demonstrations of airborne technology and DAG-TM operations. Many organizations involved in the development of new operational concepts and/or technologies for ATM have found field testing invaluable for their programs. These organizations include NASA and FAA in the development of the Center/TRACON Automation System (CTAS), ${ }^{13}$ FAA and Cargo Airline Association (CAA) in their support of the Ohio Valley Experiment, ${ }^{14}$ and Eurocontrol in the Free-Route Experimental Encounter Resolution (FREER) experiments. ${ }^{15}$ As the DAG-TM concept definition and airborne decision-aiding automation mature, sensitivity analysis will be performed on the basic assumptions embedded within the simulations. Flight test will be used to validate those data and assumptions that are critical to the proof of basic concept feasibility. Operational performance assessment and demonstrations of airborne DAG TM technology will also be accomplished.

\section{Airborne Automation Development}

Current flight management systems (FMS) provide the capability to optimize aircraft performance for a given flight plan. Additions and enhancements to existing FMS capabilities are needed for DAG TM operations. While the passive display of certain types of information, such as nearby traffic, is beneficial to the flight crew, an interactive decision aid will almost certainly be needed for real-time flight management that accounts for complex traffic and airspace constraints, schedule requirements, and other user-defined goals. Automation provides solution advisories and interactive tools to assist the crew in generating alternative solutions. Although supported by on-board automation, the crew will have final authority in all flightmanagement decisions.

NASA will develop a prototype software FMS that provides the additional functions and FMS enhancements for autonomous operations. The set of new functions will be referred to as the Autonomous Operations Planner (AOP). This planner will be a separate module within the FMS and will be generic in that it contains no aircraft performance information, allowing the software to be applicable to virtually any aircraft type. Although most functions identified for inclusion in the AOP are projected to be necessary for autonomous operations, the requirement for some functions will be dependent on the final concept of operations. Research investigations will determine which functions are actually needed and will be used to define the overall functional requirements of the AOP.

\section{$\underline{\text { Data Fusion and Interpretation }}$}

The FMS will process a variety of information about the local operational environment for use in trajectory planning. Traffic state and intent information will be received directly from other aircraft through broadcast signals such as ADS-B and indirectly though TIS. A flight information service (FIS) will provide airspace, weather, and regulatory information. Additional operational constraints and clearances may also be received by data link communication.

The FMS will interpret and fuse the information from these various sources into appropriate formats for crew display and trajectory planning. Data confidence assessment and ambiguity resolution functions will be part of this process. Confidence assessment will be important, for example, when state-vector information on a target aircraft from ADS-B and TIS are inconsistent and a determination must be made as to which data to use for display and trajectory planning.

Ambiguity resolution may be necessary where the statevector information does not match the intended trajectory as broadcast in the ADS-B message set. As one possible solution for ambiguity of intent, the AOP will include a function for inferring pilot intent by analysis of the aircraft's flight path with respect to its broadcast near-term intent, such as the next several trajectory-change points, and knowledge of the local airspace environment. Intent inferencing capability might not be necessary if all aircraft were to broadcast accurate near-term projected flight paths, faithfully follow these paths, and maintain required navigation performance (RNP) standards. However, it is unlikely that these conditions could be reliably and consistently 
met in a complex air traffic environment.

Circumstances could arise that may result in ambiguous intent information, such as failure of an aircraft's ADS$\mathrm{B}$ hardware, deviations from planned paths in response to emergencies, navigation system degradations or failures leading to loss of RNP, or the broadcast of inconsistent aircraft position and path information.

\section{Crew Interface and Display}

The crew will need the capability to supplement the automated conflict-resolution process with occasional provisional planning, i.e., manual "what-if" manipulation of user-modified (MOD) routes to meet the preferences or goals of the flight crew or company. The capability of graphical manipulation of routes has been shown to significantly improve the provisional planning process. ${ }^{16}$ Therefore, a graphical user interface (GUI) will be designed and integrated into the aircraft systems to complement the Control Display Unit (CDU). This current FMS interface permits keypad and data-link entry of information.

The GUI will likely be integrated with the navigation display to present traffic position and intent, airspace constraints, conflict information, automated resolutionadvisories, provisional planning data and other pertinent information. This display will be implemented in a human-centered design with versatile de-cluttering features. A textual interface will be retained for many of the traditional FMS/crew interface requirements and for entry of certain additional constraint and preference information related to DAG-TM operations. An additional graphical display may also be needed for long-term strategic route planning.

\section{$\underline{\text { Trajectory Generation Management }}$}

A trajectory generation management (TGM) function in the AOP will generate lateral, vertical, speed, and time constraints using calls to the hazard detection, hazard resolution, and trajectory optimization functions. The FMS flight planning and performance management functions then use these constraints to generate a flyable trajectory through iteration with the TGM. The criteria for these constraints include:

- a conflict-free path for a duration of time into the future (to be determined through research)

- airspace hazards

- regulatory position and speed restrictions

- ground-issued flow-management restrictions of position, speed, and RTA

- company passenger-comfort preferences (e.g., turbulence avoidance, minimum maneuvering)

- company business goals (e.g., preferred routes, fuel efficiency, schedule conformance)
- $\quad$ provisional planning and other preference inputs

- aircraft performance limitations

\section{Hazard Detection}

The TGM function continually analyzes the active route for hazards up to 30 minutes in the future through a hazard detection function while checking for new inputs from the flight crew or ground-based traffic

management. In addition to traffic conflicts, the hazard detection function will also detect encounters with airspace constraints.

The hazard detection function will be dependent on the ability to accurately predict target-aircraft trajectories. Accurate trajectory prediction may be required to keep spacing buffers to a reasonable size, especially for target aircraft in climbs and descents. The factors that affect accurate trajectory prediction include the ability of FMS equipped and non-FMS equipped aircraft to adhere to stated or assigned 4D trajectories, wind-field prediction, and intent knowledge. Prediction algorithms will use both state-vector projections and intent information as appropriate. Intent information provided by broadcast may range from a complete trajectory specification to few or no trajectory-change points, depending on aircraft equipage and broadcast bandwidth limitations.

Performance models may be necessary to accurately predict trajectories of target aircraft that do not broadcast sufficient information. However, the use of performance models has several disadvantages. A model may be required for every aircraft type to be encountered, and model development and distribution may require access to proprietary information. Therefore, the tradeoff between accurate trajectory prediction and inefficient airspace utilization will be examined, and performance models will be used only if determined to be essential.

\section{Hazard Resolution}

The TGM function will select a hazard resolution strategy consistent with user flight management goals and possibly the capabilities of other involved aircraft. As shown in Figure 3, the method of resolution will be dependent on the time available. For conflicts detected while in the cooperative resolution zone, the opportunity for flexibility in resolution strategies exists. These strategies can be based on user goals and mutually beneficial or agreed-to solutions between the aircraft. Having the capability to plan this far in advance and implement preferred solutions may provide one of the largest benefits of free flight to the user community, the flexibility to make business decisions in real-time flight planning. For more proximate conflicts detected within the procedural resolution zone, aircraft may be required 
to abide by flight rules that govern right-of-way and resolution maneuvers. For near-term conflicts detected within the separation assurance zone, safety may dictate a resolution strategy where the entire range of safe maneuver regions is presented to the crew.

\section{Trajectory Optimization}

For situations where the conflict is detected early enough to permit determination of a user-preferred resolution strategy, the TGM will initiate a trajectory optimization function. This function will have access to flight crew and AOC goals and preferences, TIS and FIS information, and other pertinent constraints on the flight plan. A resolution strategy that improves or optimizes the solution based on any number of optimization criteria will be determined. Optimization criteria may include minimum fuel burn, optimal arrival time, minimum flight-crew workload, and minimum passenger discomfort.

\section{Intelligent Agent Technology}

For very complex traffic situations, intelligent-agent automation may be required to support the functions of the airborne and ground-based decision aids. The automation will facilitate situational awareness by determining importance of incoming information and prioritizing the alerting of predicted events. It will predict situation complexity and provide strategies to decision makers for managing or reducing the impending workload. The automation will analyze target-aircraft actions and intent broadcasts to determine if they are contradictory. The automation will also analyze the actions of the pilot or controller and provide advisories compatible with his/her planning and preferences, using a model of goals and intent.

Research will determine the need for incorporating intelligent-agent technology into the decision aids. Significant time and effort are anticipated to bring this technology to maturity, and therefore NASA is currently initiating its development for feasibility assessment.

\section{$\underline{\text { FMS Functional Enhancements }}$}

The development of new functional capability for autonomous airborne flight operations includes the requirement for enhancements to the current FMS. Ultimately, the AOP will need to supply a hazard-free route, accounting for crew and company objectives and preferences and conforming to all externally imposed constraints and performance limitations, to the FMS guidance function. However, the current FMS has functions that are appropriate for use by the AOP in calculating the detailed trajectory from a set of given constraints. Most current FMS designs include the primary functions of flight planning, performance management, and guidance, among other functions. Enhancements or modifications to each of these functions will enable automated hazard-free route planning or provide certain functional capabilities that may influence the feasibility of DAG-TM operations. NASA will develop these capabilities so that their necessity and design requirements may be explored.

\section{FMS Flight Planning}

The flight planning function of the FMS will be modified to accept input from sources in addition to the current options of keypad entry and data link. The MOD route generated through manual provisional planning will be automatically entered as input to the flight planning function. For automated resolutions, the FMS flight planning function will accept lateral, vertical, speed, and temporal constraints from the TGM function in the AOP and generate a provisional route based on this information. The route will then be transferred to the performance management function for analysis.

\section{FMS Performance Management}

The FMS performance management functions of vertical navigation optimization and 4D-trajectory synthesis will be modified to accept a provisional route from the flight planning function. This route will be analyzed for the capability to actually fly the route given the current aircraft performance limitations. The function will reply to the TGM with "can do" or the specific constraints that could not be met and their limits. The performance management function will also provide information on request on the active trajectory for conflict detection. This information may include an estimate of future position uncertainty, depending on the method of conflict detection used.

Additional capabilities that may have benefit in DAG TM operations will be implemented. Examples are the ability to plan trajectories to meet multiple RTAs and the ability to implement continuous cruise climb.

\section{FMS Guidance}

Accurate prediction of conflicts will be dependent on the ability of aircraft to adhere to their intended trajectories, especially for conflicts involving aircraft in climb and descent phases of flight. Trajectory adherence tolerance will be determined through research. In support of this research, the guidance function will be enhanced to provide continuous closedloop temporal and spatial guidance commands to the auto-flight and auto-throttle systems. 


\section{$\underline{\text { Conclusions }}$}

Distributed air/ground traffic separation and management may help to achieve the full potential of free flight by enabling user collaboration and cooperation in all levels of traffic management decision making, thereby meeting user needs to a much greater extent than is possible today. Because DAG TM defines major changes regarding who makes decisions and who bears the costs, a policy debate will be necessary to determine whether it is in the national interest to reduce the current full reliance on centralized, ground-based air traffic management. Although the debate has already started among the various NAS stakeholders and others around the world, such decisions regarding national policy cannot be made without further information. NASA is investigating the feasibility of DAG TM through study and development of concepts and technology that distribute capability among users and service providers, using all system participants as both information suppliers and users.

\section{$\underline{\text { References }}$}

$1 \quad$ Final Report of the RTCA Task Force 3: Free Flight Implementation. RTCA, Inc., Washington DC, October 1995.

2 Duong, V. N.: FREER: Free-Route Experimental Encounter Resolution - A Solution for the European Free Flight Implementation? Air Navigation Conference, Amsterdam, 1997.

3 Hoekstra, J. M.; van Gent, R.N.H.W.; and Ruigrok, R.C.J.: Conceptual Design of Free Flight with Airborne Separation Assurance. American Institute of Aeronautics and Astronautics AIAA-98-4239, August 1998.

4 Wickens, C. D.; Mavor, A. S.; and McGee, J. P., ed.: Flight to the Future: Human Factors in Air Traffic Control. National Academy Press, Washington, D.C., 1997.

5 Andrews, J.W. and Welch, J.D.: Workload Implications of Free Flight Concepts, 1st USA/Europe Air Traffic Management R\&D Seminar, Paris, France, June, 1997.

6 Federal Aviation Administration, National Airspace System Architecture 4.0, Department of Transportation, Federal Aviation Administration, Washington, D.C., January 1999.
7 RTCA, Inc.: Government / Industry Operational Concept for the Evolution of Free Flight. RTCA, Inc., Washington DC, August 1997.

8 RTCA, Inc.: Minimum Aviation System Performance Standards for Automatic Dependent Surveillance Broadcast (ADS-B). RTCA DO-242, February, 1998.

9 The Boeing Company: Final Documentation Report: Advanced Air Transportation Technologies Free Flight Simulation Components Development. Prepared under contract NAS2-98001, March 1999.

10 Paielli, R. A. and Erzberger, H.: Conflict Probability Estimation for Free Flight. NASA TM 110411, October 1996.

11 Lockheed Martin Aeronautical Systems Company: Air Traffic Management Partnership (ATMP): An Operational Concept for Free Flight - Concept Functional Description. Prepared under contract N66001-97-C-8605, March 1998.

12 Miles, E.S., Davis, P.C. and Wing, D.J.: Development of a Free-flight Simulation Infrastructure. American Institute of Aeronautics and Astronautics AIAA-99-4193, 1999.

13 Denery, D.G. and Erzberger, H., The CenterTRACON Automation System: Simulation and Field Testing. NASA TM-110366, August 1995.

14 Operational Evaluation Coordination Group: Test \& Evaluation Master Plan Phase I-Operational Evaluation. Cargo Airlines Association and Federal Aviation Administration, April 1999.

Eurocontrol: FREER-3. http://www.eurocontrol.fr/projects/freer/freer3/ Presentation/index.htm, March 1999.

16 Battiste, V. and Johnson, W. W.: Development of a Cockpit Situation Display for Free-Flight. Society of Automotive Engineers, Inc. Technical Publication 985540, 1997. 